\title{
Lateral crural overlay of the lower lateral cartilages, a technique to enhance nasal tip rotation, and reduce projection in the droopy nasal tip
}

Accepted: 2/1/2017

Dana A. Abdilkarim *

\begin{abstract}
Background and objective: Tip plasty is one of the most common feats encountered by the Aesthetic Rhinoplasty Surgeon. Tip rotation, with or without tip deprojection, is one of the challenging problems that face the surgeon, with many overwhelming techniques to correct rotation and projection of the nasal tip, including, but not limited to overlapping of the lateral crural cartilages. This study aimed to assess the efficacy and reliability of the technique of the lateral crural overlay of the lower lateral cartilages in enhancing nasal tip rotation and decreasing nasal tip projection.
\end{abstract}

Methods: This prospective study included 64 patients, who underwent primary, and secondary, open Rhinoplasty, with nasal droopy tip and nasal tip true overprojection problems. They have been treated in Sulaimaniyah Hospital for Burn and Plastic Surgery, and from Private Hospitals in Sulaimaniyah city. The age range was 18-34 years. Forty nine patients were female, the rest of the patients were male. They have been treated, primarily, for the tip drooping and to a lesser extent for nasal tip overprojection, by the senior author, with Lateral Crural Cartilages Overlay. The study conducted from April 2012 to January 2016. The patients, postoperatively, were followed up for up to one year. Goode ratio and Nasofacial angle deducted from real time photographs have been used to evaluate the amount of rotation and deprojection of the nasal tip.

Results: Overlapping of the Lateral Crura of the Lower Lateral Cartilages, is a safe and reasonable technic in achieving nasal tip rotation, with or without, projection reduction, of the droopy nose. The operative time and dissection are very encouraging. Complications associated with this technic are very negligible, with high patient satisfaction rates. Only five rhinoplasty patient has been revised for the sake of tip rotation/projection, in this series of cases. No Major complications happened in the collumellar skin incision or the nasal rim infracartilagenous incisions, nor in the undermined nasal vestibular skin separated from the undersurface of the Lower Lateral Cartilages.

Conclusion: Overlapping, or overlaying, of the lateral crura of the lower lateral cartilages, is a reasonable technique to enhance tip rotation, and reduction of the tip projection, by medial crural steal and telescoping of the incised lower lateral cartilages, with good aesthetic and functional outcomes, with reasonable complication rates, very low revision rate, and high patient satisfaction rates.

Keywords: Lower Lateral Cartilages (LLC); Lateral Crural Overlap or overlay (LCO); Medial Crural Overlay (MCO); Nasal Tip Rotation; Nasal Tip Projection.

\section{Introduction}

Tip rotation and projection is one of the challenging problems that face the Aesthetic Plastic Surgeon, with many overwhelming techniques to correct rotation and projection of the nasal tip, including, but not limited to overlapping of the lateral crural cartilages. ${ }^{1-3}$ Overlapping of the Lower Lateral Cartilages is a novel and elegant technique for achieving nasal tip rotation and deprojection of the droopy nose, with or without some element of

* Department of Community Health / Lab Investigations, College of Health, Sulaimany Polytechnic University, Sulaimany, Iraq. 
hyperprojection. ${ }^{4,5}$ Nasal tip projection has been defined as the distance along a perpendicular line from the vertical facial plane to the most anterior projecting point of the nasal tip (Figure 1). When the nose is droopy and overprojected, it draws abnormal attention, and the normal nasofacial harmony is disturbed. This overprojection can vary from a subtlety noticed by peers, only on close analysis, to a grossly overprojected "Pinocchio" nose. ${ }^{5}$ Structural factors of the nose anatomy that produces tip over-projection may include 1- over-elongated alar cartilages, including the lateral crura, medial and middle crura or a combination of these. 2- A tense nose with gross quadrangular cartilage or nasal septum. 3- A combination of these factors and 4- Trauma or iatrogenic injury. ${ }^{1,5}$ In the classic nasal tripod theory, a standard way to effect rotation, and retrodisplacement of the tip, is to shorten one or both of the legs of the tripod. However, if one shortens only one of the legs, a change in rotation will ensue. One can take advantage of this principle to accomplish retrodisplacement and a change in rotation by selecting the proper technique. ${ }^{1.5}$ Kridel and Konior in
1989 showed that when overprojection is accompanied by tip ptosis, LCO (which shortens the lower lateral crural leg) permits incremental retrodisplacement with increased rotation. On the other hand, MCO, which shortens the medial crural leg of the tripod, leads to controlled deprojection and decreased rotation. When used together at the same surgical intervention (to shorten both tripod legs), MCO and LCO can affect large amounts of retrodisplacement with little effect on rotation. These two techniques, alone or in combination, accomplish the needed retrodisplacement in most patients. It is relatively uncommon to need further deprojection than can be accomplished with MCO or LCO alone. ${ }^{1,5}$ Many surgical technics, ${ }^{5}$ have been described for reducing the projection of the tip of the nose, including but not limited to, the medial sliding technic, ${ }^{6}$ overlapping of the lateral crus of the lower lateral cartilages, ${ }^{1-3}$ alar setback, ${ }^{7}$ Lateral Crural Setback With Cephalic Turn-in Flap, ${ }^{8}$ Hockey stick Nasal Dome Division, ${ }^{9}$, Nasal Base, Alar Base Excision Technique, ${ }^{10}$ Intermediate Crural Overlay, ${ }^{11}$ Modified Vertical Dome Division

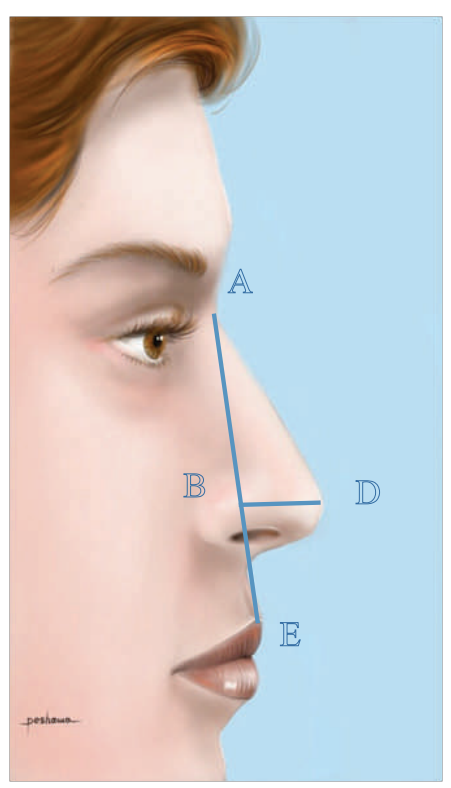

Figure 1: Illustration showing ideal nasal tip projection as deducted by analysis, defined a typical ratio equal to 0.2833 using the distance from nasion to upper vermilion-cutaneous junction of the upper lip (AE) compared with the distance of a perpendicular from this line to the tip-defining point (BD). 
Technique ${ }^{12}$ the lateral crural stairstep technique, ${ }^{13}$ and many others. ${ }^{3-19}$ Michael et al. ${ }^{14}$ recommends an incremental approach to address the nasal tip projection reduction by addressing the caudal or dorsal septum, followed by the medial crural incision or excision, and lastly by addressing the lateral crural incision or overlaying. They strongly oppose the truncation of the nasal tip cartilages because of the severe contour deformity that follows such procedures. Tongue-inGroove, TIG, Setback of the Medial crura ${ }^{15}$ by Datema FR (2014) is just another method that adds to the armamentarium of tip projection reduction. ${ }^{16-18,21-22}$ This study aimed to assess the efficacy and reliability of the technique of Lateral crural overlay of the lower lateral cartilages in enhancing nasal tip rotation and decreasing nasal tip projection.

\section{Methods}

This prospective study was performed on 64 patients who underwent Primary and Secondary Open Rhinoplasty, with droopy nasal tip, and with projection problems. They have been treated in Sulaimaniyah Hospital for Burn and Plastic Surgery and from Private Hospitals in Sulaimaniyah city. They have been treated for the tip drooping and overprojection, with Lateral Crural cartilages Overlay, (LCO). The study conducted from April 2012 to January 2016. The patients, postoperatively, followed up for up to one year. Goode ratio and Nasofacial angle deducted from real time photographs used to evaluate the amount of deprojection and rotation of the tip. Only when true nasal tip ptosis and overprojection is properly determined. Then, analysis has been done objectively. I excluded false over-projection due to low radix from this study. Infracartilagenous incisions are made on both sides and combined to an inverted $\mathrm{V}$ transcolumellar incision in the middle. The nasal skin is dissected off the alar cartilages, i.e., lower lateral cartilages in the supraperichondrial (sub SMAS, Subcutaneous Muscular
Aponeurotic System) avascular plane, or immediately above the bare cartilaginous framework of the nasal tip, up to the radix. Cephalic trimming of the lower lateral cartilages, leaving at least a 5-mm-wide strip, is done to enhance nasal tip refinement. Before making the Lateral crural cartilage incisions, the vestibular skin is undermined from the overlying lateral crus for approximately $5 \mathrm{~mm}$ on each side of the planned rotation point. The incisions in the repositioned lateral crura are then planned so as to cross the central-lateral portion of each lateral crus, comparable to Peyman, ${ }^{5}$ or the incision done in the junction of the lateral third with the medial two thirds of the overdeveloped lateral crus, comparable to Hossam. ${ }^{1}$ The cartilage incision is performed as a straight -line cephalocaudally, but care exercised to stay at least $1 \mathrm{~cm}$ beyond the domes. Dissection to release the vestibular skin also releases tethering forces that could restrict tip rotation, and it allows for safe transcartilaginous suture fixation. The integrity of the divided lateral crura is then reestablished with 5-0 PDS absorbable transcartilaginous, horizontal mattress sutures (Figure 2).

\section{Postoperative care}

The patient is encouraged to lie 45 degrees head up postoperatively for at least one week. The endonasal greasy gauze plugs removed three days postoperatively. The external nasal splints were replaced after one week by a new one, for another week. The thermal plastic split was used for this purpose. Systemic antibiotics have been used for five days after the surgery, two days parenteral and three days oral, amoxicillin-clavulanic acid. Tapping of the nose continues for at least two weeks after removal of the plastic external cast. The patient is strictly discouraged for massaging the nasal tips and the rest of the nose, in the complete rhinoplasty cases. Patients are warned about any possible trauma to the nose. Endonasal isotonic saline pump is routinely prescribed for the patients for keeping 
nasal hygiene. Transcollumellar and internal infracartilagenous incision sutures were removed completely one week. Statistical analysis applied for age range, sex distribution, frequency and percentage of complications.

Ethical considerations: Written and verbal informed consent is taken from patients before surgery. Consent was taken from the patients to use facial photos. No commercial or financial interest in any surgical material or equipment's are interested in the publishing of this paper.
Proper consent is taken from the University of Affiliation, SPU, and hospital authorities to prepare this job. No conflict of interests of any kind.

\section{Results}

The patient ages was ranging between 18 to 34 years. 49 patients $(76.5 \%)$ were female; the rest were male. The operative time (40-60 minutes) and dissection are very encouraging. No Major complications happened in the collumellar skin incision or the nasal rim infracartilagenous incisions
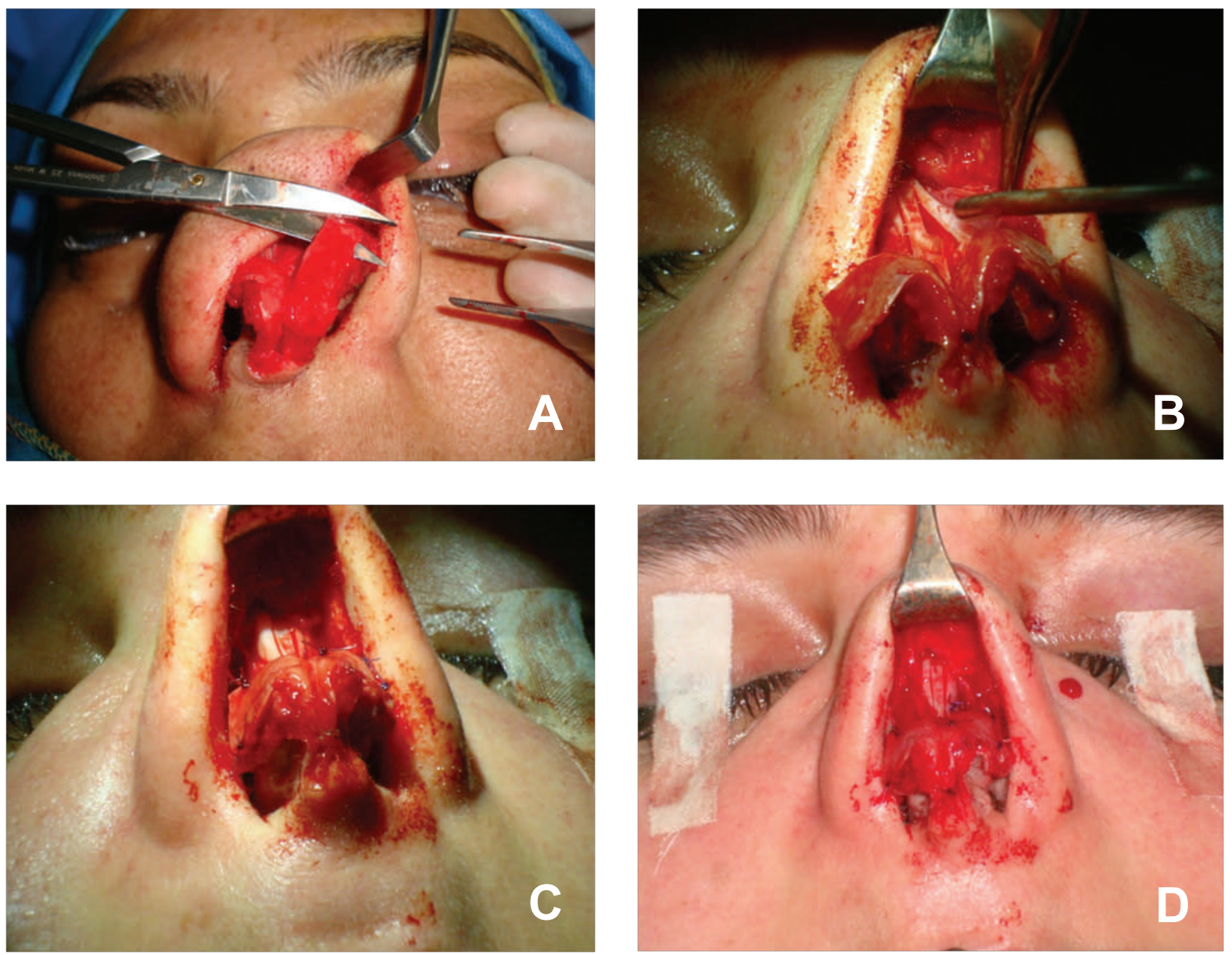

Figure 2: Surgical steps in performing lateral crural cartilage overlay $A$ and $B$ Incision of the lateral crus of the lower lateral cartilage $C$ and $D$ Fixation of the overlapped cartilages by 5/0 PDO mattress sutures 
https://doi.org/10.15218/zjms.2018.003

nor the undermined nasal vestibular skin separated from the undersurface of the LLCs (Figure 3). Minor step off in the collumellar incision in 3 cases $(4.6 \%)$ and scarring in the infracartilagenous incision sites in 2 cases (3.1\%). No projection changes noticed in 6 cases $(9.3 \%)$ although the rotation is satisfactory in the latter cases. Patients (more than $90 \%$ ) were satisfied with the net result of the tip plasties except for 6 (9.3\%) cases. Only five cases $(7.8 \%)$ were revised by alar base excision for the reason of alar base flaring. No tip necrosis, bleeding, or infection was encountered in this series of patients (Table 1).

Table 1: The frequency of complications encountered in this series of patients.

\begin{tabular}{lccc}
\hline Complications & Frequency & Percentage & \multicolumn{1}{c}{ Remarks } \\
\hline $\begin{array}{l}\text { Step off in the } \\
\text { collumellar incision }\end{array}$ & 3 & $4.6 \%$ & CO2 Laser resurfacing corrected the problems. \\
$\begin{array}{l}\text { No projection } \\
\text { changes }\end{array}$ & 6 & $9.3 \%$ & $\begin{array}{c}\text { Change in the rotation of the tip corrected the nasal } \\
\text { drooping problem. }\end{array}$ \\
$\begin{array}{l}\text { Surgical Revision } \\
\text { Alar base excision done to decrease flaring of alar base } \\
\text { and reduction of the tip projection in the cases were no } \\
\text { changes noticed in projection. These were accounting } \\
\text { for most of the patients who were not satisfied with the } \\
\text { procedure of the tip plasty. }\end{array}$ \\
$\begin{array}{l}\text { Necrosis } \\
\text { Bleeding, infection }\end{array}$ & $\begin{array}{l}7.8 \% \\
\text { nil }\end{array}$ & $\begin{array}{l}0 \% \\
0 \%\end{array}$ &
\end{tabular}

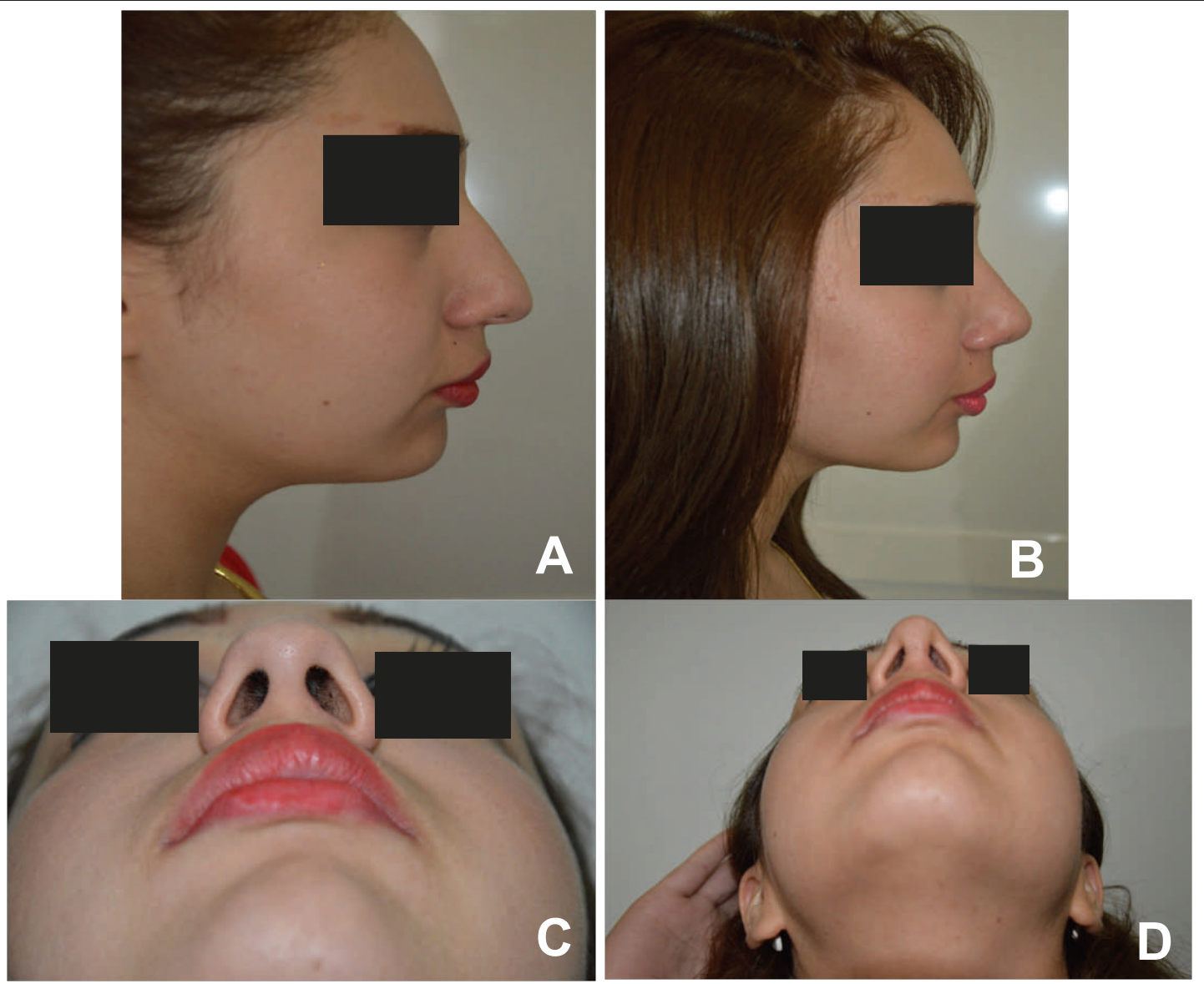

Figure 3: Case example showing LCO achieving increased rotation and depreojection, A Preop profile view B Postop profile view C Preop worm eye view D Postop worm eye view. 


\section{Discussion}

Deprojection and rotation of the overprojected nasal tip can be accomplished successfully with a handful of properly used techniques. Once the proper analysis has been accomplished, an algorithm can be used to help simplify the approach to deprojection. In a retrospective study $^{15}$ based on 22 consecutive Caucasian or Mediterranean patients who had aesthetic, open deprojection rhinoplasty combining TIG with either footplate resection or medial crural overlay by one surgeon between 2009 and 2011 . Good understanding of the anatomical and histological tip support and dynamics are very important while embarking upon any nasal tip procedure that alters projection or rotation of the nasal tip. ${ }^{19-20}$ Goode's method is based on trigonometric analyses of the whole nose. The technical construction is a little fastidious as it is based on a global analysis of the nasal dimensions. The first vertical straight line is drawn between the nasion and the alar sulcus; a second is perpendicular to the line going through the nasal tip. Methods of Calculation Goode's index is calculated as follows: 3 points are defined: B (nasion), C (nasal tip), and A (Projection of C onto the nasion-alar line). Goode's index is calculated using the ratio: $A C / B C$. A Goode's index between 0.55 and 0.6 corresponds to a normal projection. An index greater than 0.6 corresponds to hyper projection and less than 0.55 to hypo projection. When the ratio is within normal limits, it corresponds to the nasofacial angle of about $36^{\circ}$. Florence et al. (2007) present a new method of assessment of nasal tip projection, inspired by Goode's method, but which does not take into account the rotation phenomena (and thus, shortening of the dorsum). ${ }^{19}$ Regarding nasofacial angle analysis, three methods of construction were proposed by Leach to determine the naso-frontal angle: Method A measures the angle between the line of the dorsum and a vertical line connecting the glabella in the midline between the alar line and the subnasion. The dorsum line connects the nasion and the tip of the nose passing through a potential bony prominence. Method B uses as its vertical the straight line passing through the glabella and the chin. It is the most commonly used method in practice. Method C uses a vertical line which connects the nasion and the chin. ${ }^{19}$ In our series of patients, we evaluated the overlapping of the lateral crus of the lower lateral cartilages LCO with suture fixation of the medial crura to the caudal septum to reduce the nasal tip projection combined with nasal tip rotation. On reviewing the literature by Hossam, ${ }^{1}$ many of the techniques de-scribed to correct primary overprojec-tion depended on sacrifice of the domal segment of the alar cartilage by directly excising the domes. Al-though these techniques resulted in ef-fective deprojection, they were associ-ated with a high risk of tip contour irregularities in the form of pinching, notch-ing, or bossa formation, as the transected and weakened cartilages were liable to dis-placement and distortion during the heal-ing phase. This made many authors endorse domal preservation by describing alterna-tive techniques, for alar cartilage reduc-tion, depending on shortening the medial crura, lateral crura, or both. In the current study, I found that the LCO resulted in controlled predictable chang-es in the degree of tip projection as well as tip rotation. PDS absorbable sutures used in mattress fashion in contrast to authors who use permanent suture materials. ${ }^{1,5,9}$ In patients with nasal tip over-projection and caudal displacement of the tip, the freed anterior portion of the lateral crus rotates and slides over the posterior portion of the crus, which is stationary. With this overlay process; the cephalic rotation of the tip causes shortening of the lateral crura. $^{5}$ The overlapping of the lateral crura of the lower lateral cartilages is achieved through open approach rhinoplasty by proper dissection of the cutaneous skin hood of the nasal tip off the cartilaginous nasal skeleton in the 
relatively avascular loose areolar plane in between the supraperichondrial layer of the LLC just below the vestigial SMAS layer of the facial system of the subcutaneous fascia layer of the nose or just above the bare LLC. The latter is best reserved for the patients with relatively thin skin hood of the nose. No additional problem resulting from denuding the LLC is noticed, although the LLC is totally stripped from its perichondrium as the vestibular skin is dissected from the undersurface the latter cartilage to achieve the overlapping of the medial and the lateral segments of the lateral crura, to achieve the theoretical reduction of the nasal tip combined with the desired rotation of the nasal tip. On the other hand, the patients with more bulbous skin the dissection performed immediately below the SMAS since the skin thickness is sufficient to prevent noticeable step offs in the LLC. Hossam Foda ${ }^{1}$ keeps dissection of the nasal tip supraperichondrial regardless of the thickness of the nasal tip skin. Intermediate dome division, ${ }^{9,11}$ is one technic used for reduction of nasal tip projection in thin skinned individuals without causing visible deformities in the tip. Regarding planned incision of the lateral crura of the LLC, the incisions in the repositioned lateral crura are then planned so as to cross the central-lateral portion of each lateral crus, comparable to Peyman, ${ }^{5}$ or the incision done in the junction of the lateral third with the medial two thirds of the overdeveloped lateral crus, comparable to Hossam. $^{1}$ In the former technic, the cartilage cut extends in a straight line from the cephalic to the caudal crural margin, with care taken to stay at least $1 \mathrm{~cm}$ away from the dome. We found that no problem is noticed in either way, since Foda ${ }^{1}$ recommends the incision in the mentioned cartilage to be performed in the junction of the lateral third with the medial two thirds of the lateral crus because the thickness of the skin in this particular location is thick enough to camouflage any possible contour deformity resulting from overlapping of the incised margins of the lateral crus of the LLC. ${ }^{1}$ Alar base narrowing is performed as the final step in rhinoplasty because any narrowing of the nasal tip or any change in tip projection will have a direct effect on the alar base configuration. Only after the closure of all rhinoplasty incisions, the amount of alar base narrowing can be judged properly ${ }^{10}$. I was very reluctant to perform this tech to any of my patients because of the resulting scar in the alar base margin and because of difficulty in the revision of any deficiency caused by over resection in this area according to my previous experience. Hossam Foda ${ }^{1}$ in his article about LCO, has achieved very high patient satisfaction rate with very low revision $(2.6 \%)$ and complication rate. This author in another study $^{7}$ used Alar setback technique and found high patient satisfaction although the

Table 2: Some methods/Results of tip Deprojection/Rotation for comparison.

\begin{tabular}{|c|c|c|c|c|c|}
\hline Method & Results & $\begin{array}{c}\text { Patient } \\
\text { satisfaction }\end{array}$ & Revision & $\begin{array}{c}\text { Suture } \\
\text { extrusion }\end{array}$ & Other complications \\
\hline $\mathrm{LCO}^{1}$ & & High & $2.6 \%$ & zero & \\
\hline Alar setback $^{7}$ & $\begin{array}{l}8.1 \% \text { external wedge } \\
\text { excision } 6 \% \text { combined } \\
\text { with internal vestibular } \\
\text { floor excision }\end{array}$ & High & zero & $5 \%$ & $30 \%$ rim notching \\
\hline $\begin{array}{l}\text { Intermediate Crural } \\
\text { Overlay (ICO), }{ }^{11}\end{array}$ & $\begin{array}{l}\text { Rotation in } 63 \% \\
\text { Derotation in } 27 \%\end{array}$ & & $6 \%$ & & $\begin{array}{c}\text { Bossae formation in } 2 \\
\text { patients }\end{array}$ \\
\hline $\begin{array}{l}\text { Tongue-in-Groove } \\
\text { Setback of the Medial } \\
\text { Crura, }^{15}\end{array}$ & Deprojection in $86 \%$ & & & & $\begin{array}{c}\text { Increased projection } \\
\text { in } 4.5 \% \\
\text { Unchanged projection } \\
\text { in } 9.5 \%\end{array}$ \\
\hline
\end{tabular}


technique required additional maneuvers such as external wedge excision (8.1\%) and internal vestibular floor excision (6\%). Moreover, alar setback resulted in more complications. Wise et $\mathrm{al}^{11}$ deployed intermediate crural overlay technique and found $6 \%$ revision rate and bossae formation in two patients. Datema FR and Lohius $\mathrm{PJ}^{15}$ used Tongue-in-Groove setback of the medial crura and found that $4.5 \%$ had increased projection instead and $9.5 \%$ of the patients had no change in projection (Table 2 ).

\section{Conclusion}

Tip rotation and projection problems are important component of the holistic approach to the nose plastic surgery procedures Lateral crural overlapping, LCO is a safe procedure to achieve the desired rotation and projection change with functional and aesthetic results almost invariably acceptable with reasonable operative time and relative safety in performance. It decreases the need for alar base excision, with the latter procedure relatively visible additional scarring of the alar bases and the risk of alar base and nasal sill asymmetries.

\section{Competing interests}

The author declares that he has no competing interests.

\section{References}

1. Foda T. External rhinoplasty for the over projected nasal tip. English version of "Externe Rhinoplastik bei Nasenspitzenüberprojektion". J Ästhet Chir 2013; 6:26-32.

2. Foda $T$, Russell $W$, Kridel $H$. Lateral crural steal and lateral crural overlay, an objective evaluation. Arch Otolaryngology Head Neck Surg 1999; 125(12):1365-70.

3. Sajjadian A, Guyuron B. An algorithm for treatment of the drooping nose. Aesthetic Surg J 2009; 29:199-208.

4. Kridel RW, Konior RJ. Controlled nasal tip rotation via the lateral crural overlay technique. Arch Otolaryngology Head Neck Surg 1991; 117 (4):411-5.

5. Soliemanzadeh $P$, Kridel RW. Nasal Tip Overprojection, Algorithm of Surgical Deprojection Techniques and introduction of medial crural overlay. Arch Facial Plast Surg 2005; 7(6):374-80.

6. Gubisch W, Eichhorn-Sens J. The sliding technique. A method to treat the overprojected nasal tip. Aesthetic Plast Surg 2008; 32(5):7728.

7. Foda T. Alar Setback Technique, A controlled method of nasal tip deprojection. Arch Otolaryngol Head Neck Surg 2001; 127 (11):1341-6.

8. Sazgar AA. Lateral Crural Setback with Cephalic Turn-in Flap , A method to treat the drooping nose. Arch Facial Plast Surg 2010; 12(6):42730.

9. Chang CW, Simons RL. Hockey-Stick vertical dome division technique for overprojected and broad nasal tips. Arch Facial Plast Surg 2008; 10(2):88-92.

10. Foda T. Nasal Base Narrowing, The combined alar base excision technique. Arch Facial Plast Surg 2007; 9(1):30-4.

11. Wise JB, Becker SS, Sparano A, Steiger J, Becker DG. Intermediate Crural Overlay in Rhinoplasty, A deprojection technique that shortens the medial leg of the tripod without lengthening the nose. Arch Facial Plast Surg 2006; 8(4):240-4.

12. Gandomi B, Arzaghi MH, Rafatbakhsh M The effectiveness of modified vertical dome division technique in reducing nasal tip projection in rhinoplasty. Iran J Med Sci 2011; 36(3):196-200.

13. Patel KB, Mendonca DA, Skolnick G, Woo AS. Anatomical study of the medial crura and the effect on nasal tip projection in open rhinoplasty. Plast Reconstr Surg 2013; 132(4):787-93.

14. Lee MR, Geissler P, Cochran S, Gunter JP, Rohrich RJ. Decreasing Nasal Tip Projection in Rhinoplasty. Plast Reconstr Surg 2014; 134(1):41e-9.

15. Datema FR, Lohuis PJ. Tongue-in-Groove setback of the medial crura to control nasal tip deprojection in open rhinoplasty. Aesthetic Plast Surg 2014; 39(1):53-62.

16. Busca GP, Amasio ME, Staffieri A. The surgery of the tip of the nose. Acta Otorhinolaryngol Ital 2002; 22(3 Suppl 70):7-29.

17. Lewis JR. Nasal tip projection: counterpoint. Plast Reconstr Surg 1987; 80(3):356-65.

18. Han SK, Lee DG, Kim JB, Kim WK. An anatomic study of nasal tip supporting structures. Ann Plast Surg 2004; 52(2):134-9.

19. Turner F, Zanaret M, Giovanni A. Evaluation of nasal tip projection. Fr ORL 2007; 92:282-7.

20. Kridel RW, Scott BA, Foda HM. The tongue-inGroove technique in septorhinoplasty, A 10-Year Experience. Arch Facial Plast Surg 1999; 1(4):246-58. 